\title{
REFERENCES
}

1. R. Courant and D. Hilbert, Methods of mathematical physics, Vol. 1, Interscience Publishers, Inc., New York, 1953, p. 158

2. P. Hartman and A. Wintner, On the solutions of the equations of heat conduction, Amer. J. Math. 72 (1950) 367

3. F. G. Tricomi, Integral equations, Interscience Publishers, Inc., New York, 1957, p. 39

4. H. Weber, Die partiellen Differentialgleichungen der mathematischen Physik, Friedr. Vieweg und Sohn, Braunschweig, Germany, 1912, pp. 101-111

\section{A VARIATIONAL PRINCIPLE FOR NONLINEAR NETWORKS*}

\section{BY GARRETT BIRKHOFF (Harvard University)}

It is the main purpose of this note to describe a variational principle for nonlinear networks of the kind studied by J. B. Diaz and the author in [1]. It will then be shown that this principle is closely related to an electromechanical analogy discovered by Duffin [2], in connection with $n$-dimensional mass-spring networks.

The notation and terminology of [1] will be used below. That is, a connected network (oriented graph) $N$ of $n$ nodes $A_{k}$ and $r$ links $a_{i}$ will be assumed defined by an incidence matrix $\left\|\epsilon_{k i}\right\|$, where $\epsilon_{k j}$ is $+1,-1$, or 0 when $A_{k}$ is the initial node, the final node, or not incident on $a_{i j}$, respectively. In $N$, a subset $\partial N$ of boundary nodes is distinguished, and the set of all links is denoted by $L$.

It is assumed that, in equilibrium, the current $i_{j}=c_{i}\left(\Delta u_{i}\right)$ is a specified continuous increasing function $c_{j}\left(\Delta u_{j}\right)$ of the potential drop $\Delta u_{j}=\sum_{k} \epsilon_{k j} u_{j}$ across the $j$-th link. At each interior node $A_{k}$ (not on $\partial N$ ), the usual node law $\sum_{i=1}^{r} \epsilon_{k} i_{i}=0$ is assumed. At each boundary node $A_{h}$, it is assumed that either $u_{h}$ is known or that (on the residual set $\left.\partial^{*} N\right)$

$$
\sum_{i} \epsilon_{h i} i_{i}=\sum_{i} \epsilon_{h j} c_{i}\left(\sum_{k} \epsilon_{k j} u_{k}\right)=F_{h}\left(u_{h}\right) \text { on } \partial * N,
$$

where $F_{h}(u)$ is a continuous non-increasing function of $u$. (The case $F_{h}(u)=\nu_{h}$ constant leads to the Neumann problem.)

With the preceding problem, we now associate the function

$$
V=\sum_{L} V_{j}\left(\Delta u_{i}\right)+\sum_{\partial * N} W_{h}\left(u_{h}\right),
$$

where the functions $V_{i}$ and $W_{h}$ are defined as the indefinite integrals

$$
V_{i}\left(\Delta u_{i}\right)=\int_{0}^{\Delta u_{i}} c_{j}(s) d s, \quad W_{h}\left(u_{h}\right)=-\int_{0}^{u_{h}} F_{h}(s) d s .
$$

We take as unknowns the values $u_{k}=u\left(A_{k}\right)$ at those interior and boundary nodes where the potential $u_{k}$ is not given.

An easy computation shows that the first variation of the expression (2) defined by $\left(2^{\prime}\right)$ is

*Received October 11, 1962. 


$$
\delta V=\sum_{L} c_{j}\left(\Delta u_{i}\right) \sum \epsilon_{k i} \delta u_{k}-\sum_{\partial * N} F_{h}\left(u_{h}\right) \delta u_{h},
$$

where the variations $\delta u_{h}$ are independent over the nodes where $u_{h}$ is not given.

Theorem 1. The conditions for equilibrium are equivalent to the variational equation $\delta V=0$.

Proof. At a boundary node where $u_{h}$ is not given, the coefficient of $\delta u_{h}$ in (3) vanishes if and only if

$$
\sum_{L_{h}} \epsilon_{h i} i_{i}=F_{h}\left(u_{h}\right),
$$

summed over the links incident with $A_{h}$. Clearly, equations (4) and (1) are identical. The proof for internal nodes is identical.

The preceding proof makes use only of the continuity of the functions $c_{i}$ and $F_{h}$, which makes valid the differentiation of (2) to get (3). Therefore, the statement of Theorem 1 is true whether or not the $c_{i}$ are increasing and the $F_{h}$ non-increasing functions.

Theorem 2. If the functions $c_{i}$ are non-decreasing and the $F_{h}$ non-increasing, then the function $V(\mathbf{u})$ defined by $(2)-\left(2^{\prime}\right)$ is convex.

Proof. It suffices to prove that

$$
V(\mathbf{u}+\mathbf{v})+V(\mathbf{u}-\mathbf{v})-2 V(\mathbf{u}) \geqq 0 .
$$

But, referring to the definition (2)-(2'), we have

$$
\begin{aligned}
\Delta^{2} V_{i} & =V_{i}\left(\Delta u_{i}+\Delta v_{i}\right)+V_{i}\left(\Delta u_{i}-\Delta v_{i}\right)-2 V_{i}\left(\Delta u_{i}\right) \\
& =\int_{0}^{\Delta v_{i}}\left[c_{i}\left(\Delta u_{i}+s\right)-c_{i}\left(\Delta u_{i}-s\right)\right] d s \geqq 0
\end{aligned}
$$

for all $j$, provided the $c_{i}$ are non-decreasing. Likewise,

$$
\begin{aligned}
\Delta^{2} W_{h} & =W_{h}\left(u_{h}+v_{h}\right)+W_{h}\left(u_{h}-v_{h}\right)-2 W_{h}\left(u_{h}\right) \\
& =-\int_{0}^{\Delta u_{h}}\left[F_{h}\left(u_{h}+s\right)-F_{h}\left(u_{h}-s\right)\right] d s \geqq 0,
\end{aligned}
$$

provided every $F_{h}$ is non-increasing.

It is interesting that the statement of Theorem 2 is true whether or not the $c_{i}$ and the $F_{h}$ are continuous. It is also interesting to inquire into the conditions for strict convexity: that strict inequality holds in (5). Inspecting the inequalities used in proving (5), we see

Theorem 3. The function $V(\mathfrak{u})$ defined by $(2)-\left(2^{\prime}\right)$ is strictly convex if and only if the $c_{i}$ are increasing functions and the $F_{h}$ decreasing functions.

Corollary. Under the preceding hypotheses, there is at most one equilibrium solution of the nonlinear network problem, and this occurs when $V$ has a strict minimum.

For the existence of an absolute minimum to a (continuous) convex function $V(\mathbf{u})$, it is sufficient that $\lim _{|\mathbf{u}| \rightarrow \infty} V(\mathfrak{u})=+\infty$. Therefore, it is sufficient that all functions $V_{i}\left(\Delta \mathbf{u}_{i}\right)$ and $W_{h}\left(u_{h}\right)$ should tend to $+\infty$ with $\left|\Delta u_{i}\right|$, as this will make some terms in (2) tend to $+\infty$ with $|\mathbf{u}|$, while the others remain bounded below. We conclude

TheOREM 4. If the functions $c_{j}$ are non-decreasing and the $F_{h}$ non-increasing, and if these functions assume both signs, then $V$ has a minimum.

Note that, since monotone functions are always integrable, continuity is not assumed above. 
Corollary. If the $c_{j}$ and $F_{h}$ are continuous in Theorem 4 , then the network problem has a solution.

In conclusion, it may be of interest to describe the mechanical analog of the network problem, whose potential (strain) energy function is $V(\mathbf{u})$. In this analog, the nodes are represented by smooth rods, whose displacements from given positions are the $u_{i}$. These rods are constrained to slide along the $u$-axis, a constraint which can be imposed by inserting the rods in a long smooth tube. These rods can then be imagined as joined by one or more springs, for each link $a_{i}$ whose total stress $c_{j}\left(\Delta u_{i}\right)$ depends on the relative displacement of the rods joined by the link $a_{i}$. This acts equally and in opposite directions as a force on these rods, as prescribed by the $\epsilon_{k j}$ (thus stress or force is the mechanical analog of current). At internal nodes, the condition for equilibrium is that the sum (resultant) of the forces be zero. At boundary nodes, one can suppose externally attached springs exert a force $F_{h}$ which depends on the displacement of the $\operatorname{rod} A_{h}$.

This model is very similar to that suggested by Duffin [2], provided the junction points are constrained to move in one dimension. However, Duffin's "force functions" $f_{i j}\left(\left|\mathbf{r}_{i}-\mathbf{r}_{i}\right|\right)$ were assumed to be defined only for non-negative arguments, to satisfy $f(0)=0$, and to be unbounded. This would correspond roughly to assuming the $c_{j}\left(\Delta u_{j}\right)$ to be odd functions tending to infinity with $\left|\Delta u_{i}\right|$, and these assumptions seem to be unnecessary for the existence and uniqueness theorems stated above. Duffin also considers only the Dirichlet problem (all $F_{h}$ vanish).

Duffin's hypotheses are, of course, appropriate for networks of springs under tension in more than one dimension.

\section{REFERENCES}

1. G. Birkhoff and J. B. Diaz, Non-linear network problems, Q. Appl. Math. 13 (1956) 431

2. R. J. Duffin. Non-linear networks IIA, Bull. Am. Math. Soc. 53 (1947) 963

\section{AN INTEGRAL EQUATION OCCURRING IN PLASMA OSCILLATIONS*}

By R. S. B. ONG (The University of Michigan)

The stability of single hump velocity distributions in a collisionless hot plasma without magnetic field is usually shown by considering the linearized Landau-Vlasov self-consistent set of equations as an initial value problem. The Laplace-or the onesided Fourier transform with respect to the time variable is then commonly used in the analysis. In this method, one needs the analytic continuation of the function considered beyond its original domain of definition in order to evaluate its inverse transform by complex contours. This introduces a certain degree of artificiality in the analysis. The purpose of this article is to show that one can deduce the stability of single hump velocity distributions by means of an argument which avoids this difficulty.

The one-dimensional linearized problem of longitudinal oscillations of a collisionless hot plasma without an external magnetic field is described by the following well-known self-consistent set of equations:

\footnotetext{
* Received November 5, 1962. This work was performed with the aid of a grant from the National Aeronautics and Space Administration.
} 